\title{
INTEGRAL REPRESENTATION IN THE HODOGRAPH PLANE OF COMPRESSIBLE FLOW
}

\author{
BY \\ ERIK B. HANSEN (Department of Mathematics, Technical University of Denmark, DK 2800, \\ Lyngby, Denmark) \\ AND
}

GEORGE C. HSIAO (Department of Mathematical Sciences, University of Delaware, Newark, Delaware 19716, USA)

\begin{abstract}
Compressible flow is considered in the hodograph plane. The linearity of the equation determining the stream function is exploited to derive a representation formula involving boundary data only, and a fundamental solution to the adjoint equation. For subsonic flow, an efficient algorithm for computing the fundamental solution is developed.
\end{abstract}

1. Introduction. Integral equation methods have been used extensively in fluid mechanics as reviewed by Morino [1] and Pozrikidis [2]. By far, the majority of the applications have considered inviscid, incompressible potential flow or Stokes' flow, where the governing equations are linear, thereby allowing one of the main advantages of integral equation methods: the reduction of the dimension of the problem by one unit. Integral equation methods have also been applied to compressible, including transsonic, flow, even though the equations for compressible flow in the physical plane are nonlinear. A pioneer in this field was Ostwatitsch [3]. Early applications to compressible flows were presented in [4] and [5], and more recent ones are reviewed in [1]. They are all based directly on the equations in the physical plane. If the equations are not linearized, the nonlinear terms give rise to domain integrals in the representation formula, so that a reduction of dimension is not achieved until further, simplifying approximations, besides the fundamental ones of irrotational and homentropic flow, are made.

Most of the work referred to above deals with two-dimensional problems, and it is well known that in two dimensions the equations for compressible flow become linear when transformed to the hodograph plane. Because of this property, the hodograph transformation has often been applied to compressible flow problems. An early application of the hodograph transformation was made by Lighthill in three papers [6], [7], and [8], on flow in a symmetric channel or around a cylindrical body. Several results on the mathematical

Received February 21, 2000.

2000 Mathematics Subject Classification. Primary 35C15, 76G25, 76M15. 
properties of the transformation are also presented in these papers. Other applications to specific problems are found in [4] and [5], and in [9] the hodograph transformation is used in a theoretical investigation. However, surprisingly, despite the fact that an integral equation formulation of a compressible flow problem in the hodograph plane would lead to a boundary integral equation and, consequently, to a reduction of dimension by one, this avenue does not seem to have been investigated in the literature.

The purpose of this paper is to develop tools which are necessary for solving compressible flow problems via boundary integral methods in the hodograph plane. The focus is on subsonic problems. As we shall see, since the fluid velocity at a rigid body surface is not known a priori, a boundary value problem with fixed boundaries in the physical plane becomes a free boundary problem in the hodograph plane. However, integral equation methods are also well suited for solving free boundary problems and have often been used for that purpose, e.g., in [10] and [11], so this is not a serious drawback.

The rest of this paper is organized as follows. In Sec. 2 we review, for later reference, the equations for compressible flow in the physical as well as in the hodograph plane, and we formulate a typical, subsonic boundary value problem in the hodograph plane. One of the two main tools of integral equation formulations of boundary value problems is a representation formula for solutions to the partial differential equation. In Sec. 3 we derive one for the equation determining the stream function. The other main tool, a fundamental solution to the adjoint equation, is found in Sec. 4, and in Sec. 5 we describe its numerical evaluation in the subsonic region. Work on the applications to specific problems is in progress, and the extension to the supersonic region is being investigated.

2. Boundary value problems in the hodograph plane. The equation of motion for a steady, two-dimensional, compressible flow of an inviscid fluid is

$$
\rho\left(u u_{x}+v u_{y}\right)+p_{x}=0 \text { and } \rho\left(u v_{x}+v v_{y}\right)+p_{y}=0,
$$

and the continuity equation is

$$
(\rho u)_{x}+(\rho v)_{y}=0 .
$$

Here $\rho$ is the density, $p$ is the pressure, and $(u, v)$ is the velocity referred to a rectangular $x y$-coordinate system. We shall treat the fluid as an ideal gas and assume that the flow is irrotational and homentropic. Then,

$$
u_{y}=v_{x}
$$

and the ratio

$$
\frac{p}{\rho^{\gamma}}=\frac{p_{0}}{\rho_{0}^{\gamma}}
$$

is a global constant in the fluid region. The quantities $p_{0}$ and $\rho_{0}$ introduced here shall denote the values of $p$ and $\rho$, respectively, at a stagnation point. The exponent $\gamma$ in (4) is Poisson's ratio between the specific heats at constant pressure and volume, respectively. For air, $\gamma=1.41$. 
The speed of sound in an ideal gas is equal to

$$
c=\sqrt{\frac{d p}{d \rho}}=\sqrt{\gamma \frac{p}{\rho}}
$$

From (4) and (5), it is seen that $p$ and $\rho$ can be expressed as

$$
p=p_{0}\left(\frac{c}{c_{0}}\right)^{\frac{2 \gamma}{\gamma-1}} \text { and } \rho=\rho_{0}\left(\frac{c}{c_{0}}\right)^{\frac{2}{\gamma-1}}
$$

where $c_{0}$ is the speed of sound at a stagnation point. It follows from (1), (2), and (5) that

$$
\left(u^{2}-c^{2}\right) u_{x}+u v\left(u_{y}+v_{x}\right)+\left(v^{2}-c^{2}\right) v_{y}=0 .
$$

Application of (3) and (6) in (1) leads to Bernoulli's equation:

$$
c^{2}=c_{0}^{2}-\frac{(\gamma-1)}{2} q^{2}
$$

where $q=\left(u^{2}+v^{2}\right)^{1 / 2}$. Since $c$ cannot be negative, Eq. (8) shows that the mathematical description of a flow based on (1), (2), (3), and (4) implies that $q$ cannot exceed the value

$$
q_{\max }=\sqrt{\frac{2}{\gamma-1}} c_{0}
$$

We also see that $c=q$ at the so-called critical speed,

$$
q_{\text {crit }}=\sqrt{\frac{2}{\gamma+1}} c_{0} \text {. }
$$

Therefore a flow is called subsonic at a point if $q<q_{\mathrm{crit}}$, and supersonic at a point if $q>q_{\text {crit }}$.

The continuity equation implies that in a simply connected region, $\rho u$ and $\rho v$ can be expressed in terms of a stream function, $\psi$, as

$$
\rho u=\psi_{y} \quad \text { and } \quad \rho v=-\psi_{x} .
$$

The same is true for flows in multiply-connected regions, if the surfaces of the imbedded obstacles are impermeable.

To set up an equation and boundary conditions for $\psi$ in the hodograph plane we first express $\psi$ in (11) as a function of $u$ and $v$ :

$$
\rho u=\psi_{u} u_{y}+\psi_{v} v_{y} \quad \text { and } \quad \rho v=-\psi_{u} u_{x}-\psi_{v} v_{x} .
$$

From these relations, (3), and (7), we find expressions for $u_{x}, u_{y}, v_{x}$, and $v_{y}$ in terms of $\psi_{u}$ and $\psi_{v}$, which we use to derive the formulae:

$$
q_{x}=-D \psi_{v} \quad \text { and } \quad q_{y}=D \psi_{u},
$$

where

$$
D=\frac{\rho q c^{2}}{\left(c^{2}-v^{2}\right) \psi_{u}^{2}+2 u v \psi_{u} \psi_{v}+\left(c^{2}-u^{2}\right) \psi_{v}^{2}} .
$$

When $q_{x}$ and $q_{y}$ are eliminated from (13) by cross differentiation, the following equation is obtained:

$$
\left(1-\frac{v^{2}}{c^{2}}\right) \frac{\partial^{2} \psi}{\partial u^{2}}+\frac{2 u v}{c^{2}} \frac{\partial^{2} \psi}{\partial u \partial v}+\left(1-\frac{u^{2}}{c^{2}}\right) \frac{\partial^{2} \psi}{\partial v^{2}}+\frac{2}{c^{2}}\left(u \frac{\partial \psi}{\partial u}+v \frac{\partial \psi}{\partial v}\right)=0
$$


If the polar coordinates $\rho$ and $\theta$, defined in Fig. 1, are introduced as independent variables, then this equation becomes Chaplygin's equation [12].
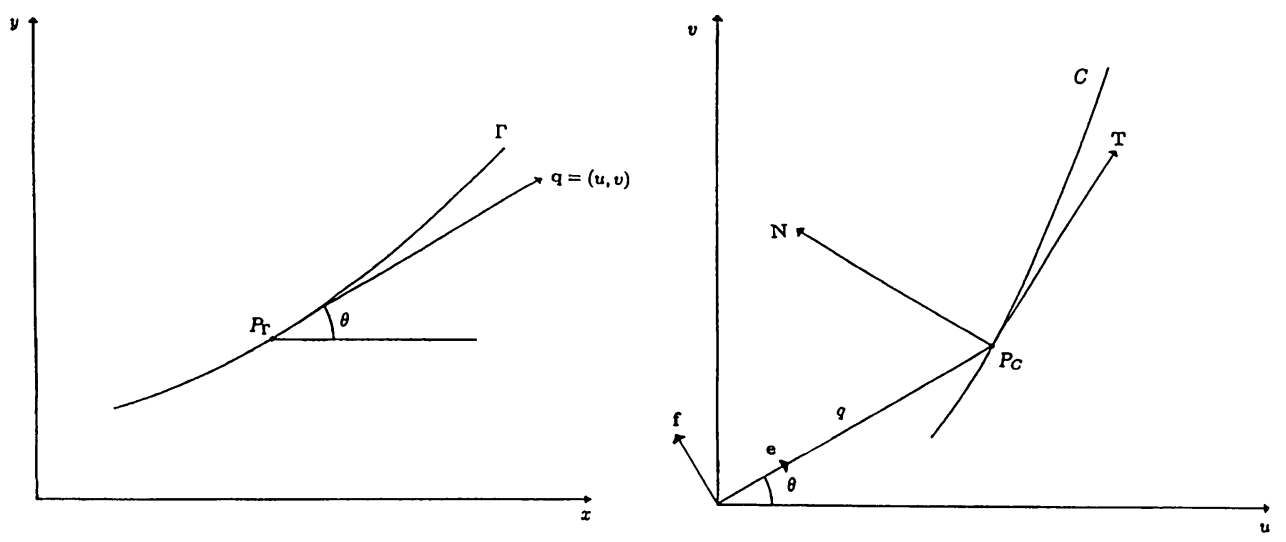

FIG. 1. The trace $\Gamma$ of a rigid cylinder in the physical plane and its image $C$ in the hodograph plane

We hereafter consider the boundary conditions for $\psi$ in the hodograph plane. Let the curve $\Gamma$ in Fig. 1 be the intersection of a rigid, impermeable cylinder with the physical plane, and let $C$ be its image in the hodograph plane. At an arbitrary point $P_{\Gamma}$ on $\Gamma$, the fluid velocity is tangential. So, the coordinate $\theta$ of the image $P_{C}$ of $P_{\Gamma}$ is equal to the angle subtended between the tangent of $\Gamma$ at $P_{\Gamma}$ and the $x$-axis. However, the fluid speed at $P_{\Gamma}$ is not known a priori, and therefore the coordinate $q$ of $P_{C}$, and hence the location of the image of $\Gamma$, is unknown. Thus, a problem with rigid boundaries in the physical plane becomes a free boundary problem when transformed to the hodograph plane, as was pointed out by Nocilla [13].

Since the stream function assumes a constant value on a rigid boundary $\Gamma$ in the physical plane, it must assume the same constant value on the image $C$ in the hodograph plane. It follows from a theorem [14] for a class of elliptic equations, which includes (15), that in an arbitrary region bounded by a piecewise differentiable curve, there exists a unique solution which assumes a given, constant value on the boundary. Thus, in order that the image $C$ can be determined, more than one boundary condition must be prescribed on $C$. In analogy with other free boundary problems with second order elliptic equations (see, e.g., [15]), we expect exactly one extra condition, which fixes the value of the normal derivative $\partial \psi / \partial N$, to be needed.

To introduce $\partial \psi / \partial N$ in the analysis, we note that if $\mathbf{T}=\left(T_{u}, T_{v}\right)$ is the unit tangent vector on $C$, the fact that $\psi$ is constant on $C$ implies that $\psi_{u} T_{u}+\psi_{v} T_{v}=0$. So, if the normal of $C$ is defined as indicated in Fig. 1:

$$
\psi_{u}=-T_{v} \frac{\partial \psi}{\partial N} \quad \text { and } \quad \psi_{v}=T_{u} \frac{\partial \psi}{\partial N},
$$

then the derivatives $q_{x}$ and $q_{y}$ in (13) and all linear combinations of $q_{x}$ and $q_{y}$ can be expressed in terms of $\partial \psi / \partial N$. The derivative $d q / d \theta$ along $\Gamma$ or $C$ is such a linear combination, and it can be expressed in a simple way in terms of variables in the physical 
plane as well as in terms of those in the hodograph plane. In the physical plane, let $l$ be the arclength along $\Gamma$ and $\kappa=\kappa(\theta)$ the curvature of $\Gamma$ defined as $\kappa=d \theta / d l$. Then:

$$
\frac{d q}{d \theta}=\frac{1}{\kappa} \frac{d \theta}{d l}=\frac{1}{\kappa}\left[q_{x} \cos \theta+q_{y} \sin \theta\right]=\frac{q_{x} u+q_{y} v}{\kappa q} .
$$

In the hodograph plane it is convenient to express $d q / d \theta$ in terms of the vector $\mathbf{q}=$ $(u, v)$ and the unit tangent and normal vectors, $\mathbf{T}$ and $\mathbf{N}$, on $C$. To do so, we write $\mathbf{T}$ as

$$
\mathbf{T}=\frac{d \mathbf{q}}{d s}=\frac{d q}{d s} \mathbf{e}+q \frac{d \mathbf{e}}{d s}=\frac{d q}{d s} \mathbf{e}+q \frac{d \theta}{d s} \mathbf{f} .
$$

Here $s$ is the arclength along $C$, and $\mathbf{e}$ and $\mathbf{f}$ are unit vectors parallel with and perpendicular to q, respectively (see Fig. 1). Taking the scalar product of the two sides with $\mathbf{N}$, we get that

$$
0=\frac{d q}{d s} \mathbf{e} \cdot \mathbf{N}+q \frac{d \theta}{d s} \mathbf{f} \cdot \mathbf{N}=\frac{d q}{d s} \frac{\mathbf{q} \cdot \mathbf{N}}{q}+\frac{d \theta}{d s} \mathbf{q} \cdot \mathbf{T} .
$$

In (17) we now insert $q_{x}$ and $q_{y}$ from (13), where $\psi_{u}$ and $\psi_{v}$ are expressed as in (16), and replace $d q / d \theta$ by the expression determined from (19). Thereby the following formula is obtained:

$$
\frac{\partial \psi}{\partial N}=\frac{\rho c^{2} \mathbf{N} \cdot \mathbf{q}}{\kappa(\theta)\left[c^{2}-(\mathbf{T} \cdot \mathbf{q})^{2}\right]} .
$$

This formula is equivalent to one derived earlier by Nocilla [13].

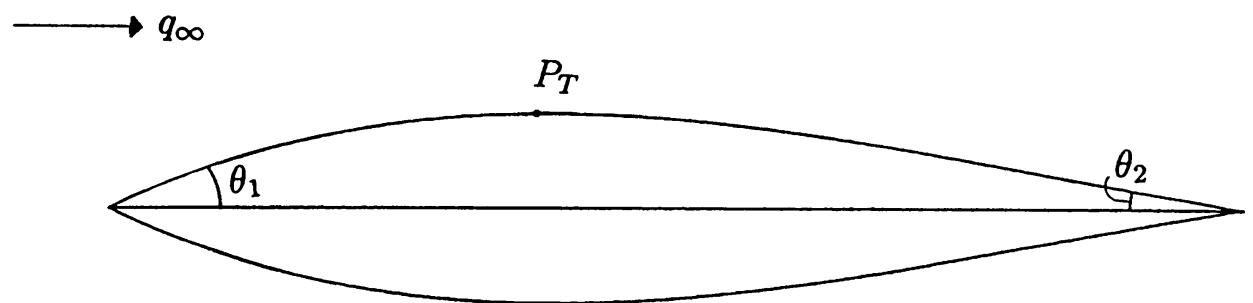

FIG. 2. A cylindrical, symmetrical airfoil is placed in a uniform flow with speed $q_{\infty}$, so that the symmetry axis is parallel to the undisturbed fluid velocity

We are now in a position to formulate a subsonic, compressible flow problem as a boundary value problem in the hodograph plane. As a simple example we consider the flow around a cylindrical, symmetric airfoil, which is introduced in a uniform flow with speed $q_{\infty}$ in such a way that its symmetry axis is parallel to the undisturbed flow direction (see Fig. 2). On the symmetry axis upstream from the airfoil, the velocity is parallel to the $x$-axis and the speed decreases from $q_{\infty}$ at infinity to 0 at the leading edge of the airfoil. (The airfoil is assumed not to have cusps at its edges.) Therefore the image of the upstream part of the symmetry axis is the line section $C_{S}=\left\{\theta, q \mid \theta=0, \quad 0<q<q_{\infty}\right\}$. On the upper side of the airfoil, the velocity at the leading edge points in the direction $\theta=\theta_{1}$. The direction of the velocity turns clockwise, as the upper half of the contour is transversed, and points in the direction $\theta=-\theta_{2}$ at the trailing edge, where $q=0$. So, the image of the upper half of the contour is a curve, $C_{A}$, through the origin with tangent direction $\theta=\theta_{1}$ and $\theta=-\theta_{2}$ at that point, as indicated in Fig. 3. The image of the top 
point $P_{T}$ on the airfoil is the intersection of $C_{A}$ with the $u$-axis. On the symmetry line downstream from the trailing edge, the velocity increases from 0 to $q_{\infty}$. That part of the symmetry line, too, is therefore mapped onto the line section $C_{S}$. Thus, the image of the flow field in the upper half of the physical plane is the region bounded by $C_{A}$ and cut along $C_{S}$. On $C_{S}$ and on $C_{A}, \psi$ assumes one and the same constant value. On $C_{A}$, $\partial \psi / \partial N$ is given by $(20)$.

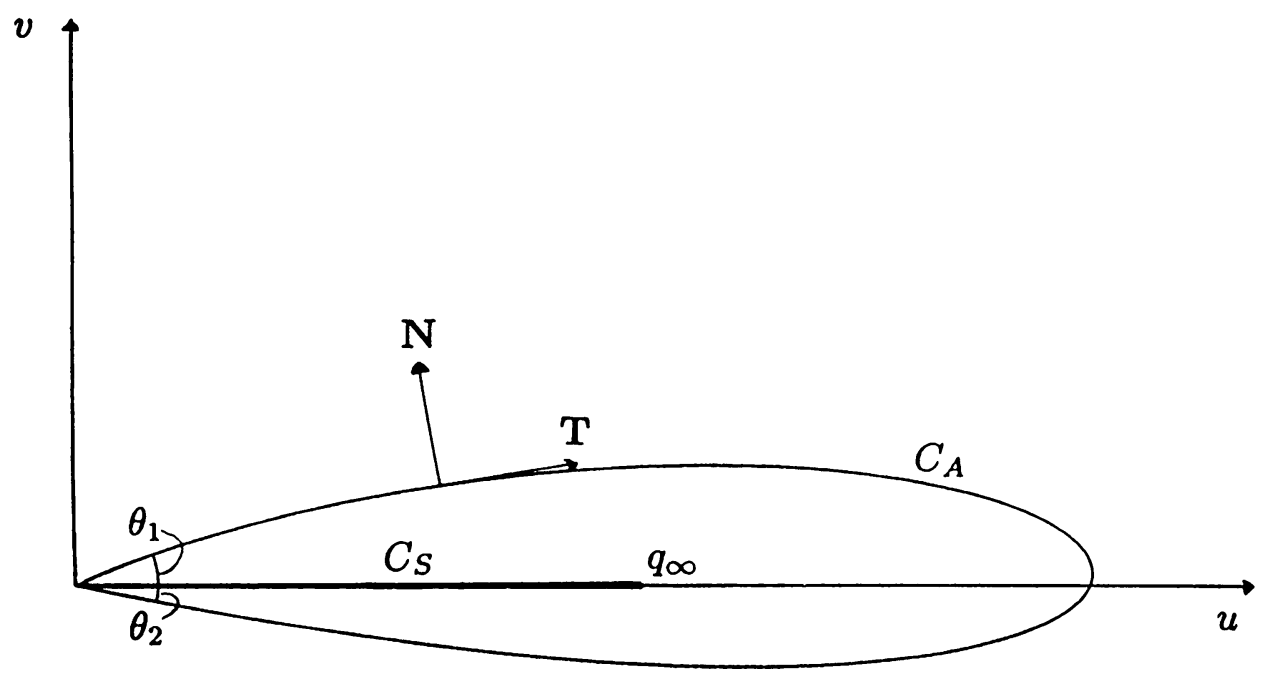

FIG. 3. The image of upper half of the airfoil contour in Fig. 2 is the curve $C_{A}$, and the image of the flow region above the symmetry axis of the airfoil is the region bounded by $C_{A}$ and cut along the line section $C_{S}$ on the $u$-axis from 0 to $q_{\infty}$

3. The representation formula. To derive a representation formula for solutions to Eq. (14), we consider the integral

$$
\int_{\Omega} G L \psi d \Omega=0
$$

The domain of integration $\Omega$ is a bounded region in the hodograph plane, $G$ is a fundamental solution to an equation to be chosen later, $L \psi$ denotes the left-hand side of Eq. (15), and $\psi$ is a solution to Eq. (15) everywhere in $\Omega$. Therefore the integral vanishes.

All expressions in the following derivation of the representation formula are to be interpreted in the sense of distribution theory.

To derive a form of the integrand in which there are no derivatives of $\psi$, we write the product of $G$ and the first term of $L \psi$ as follows using (8):

$$
\begin{gathered}
G\left(1-\frac{v^{2}}{c^{2}}\right) \frac{\partial^{2} \psi}{\partial u^{2}} \\
=\frac{\partial}{\partial u}\left[\left(1-\frac{v^{2}}{c^{2}}\right) G \frac{\partial \psi}{\partial u}\right]-\left(1-\frac{v^{2}}{c^{2}}\right) \frac{\partial G}{\partial u} \frac{\partial \psi}{\partial u}+\frac{u v^{2}}{c^{4}}(\gamma-1) G \frac{\partial \psi}{\partial u} .
\end{gathered}
$$


When the other terms are recast in similar ways, we find by repeated application of Gauss' formula that (21) can be written as

$$
\int_{\partial \Omega} B(G, \psi) d s+\int_{\Omega} \psi \widetilde{L} G d \Omega=0 .
$$

Here $s$ is the arclength along the boundary, $\partial \Omega$, of $\Omega$ :

$$
\begin{aligned}
B(G, \psi)= & {\left[\left(1-\frac{v^{2}}{c^{2}}\right)\left(G \frac{\partial \psi}{\partial u}-\psi \frac{\partial G}{\partial u}\right)+\frac{u v}{c^{2}}\left(G \frac{\partial \psi}{\partial v}-\psi \frac{\partial G}{\partial v}\right)+\frac{u}{c^{2}} G \psi\right] N_{u} } \\
& +\left[\left(1-\frac{u^{2}}{c^{2}}\right)\left(G \frac{\partial \psi}{\partial v}-\psi \frac{\partial G}{\partial v}\right)+\frac{u v}{c^{2}}\left(G \frac{\partial \psi}{\partial u}-\psi \frac{\partial G}{\partial u}\right)+\frac{v}{c^{2}} G \psi\right] N_{v}
\end{aligned}
$$

and $\widetilde{L}$ is the adjoint of $L$ :

$$
\widetilde{L} G=\left(1-\frac{v^{2}}{c^{2}}\right) \frac{\partial^{2} G}{\partial u^{2}}+\frac{2 u v}{c^{2}} \frac{\partial^{2} G}{\partial u \partial v}+\left(1-\frac{u^{2}}{c^{2}}\right) \frac{\partial^{2} G}{\partial v^{2}}-\frac{2 c_{0}}{c^{4}} G .
$$

We now choose $G$ to be a fundamental solution to the equation $\widetilde{L} w=0$, that is, $G=G\left(u, v, u^{\prime}, v^{\prime}\right)$, and

$$
\widetilde{L} G=-\delta\left(u-u^{\prime}\right) \delta\left(v-v^{\prime}\right)
$$

where $\delta$ is Dirac's delta function. It then follows from $(23)$ that if $\left(u^{\prime}, v^{\prime}\right)$ is a point in the interior of $\Omega$, then $\psi\left(u^{\prime}, v^{\prime}\right)$ can be expressed as

$$
\psi\left(u^{\prime}, v^{\prime}\right)=\int_{\partial \Omega} B(G, \psi) d s
$$

If $\left(u^{\prime}, v^{\prime}\right)$ is neither in the interior of $\Omega$ nor on the boundary $\partial \Omega$, then the integral in (27) vanishes. Formula (27) is a representation for solutions to the equation for the stream function in the hodograph plane.

Since the boundary conditions in the hodograph plane are conveniently expressed in terms of the normal and tangential derivatives of $\psi$, we choose to write the integrand $B(\psi, G)$ given by $(24)$ in terms of these derivatives. If the unit tangent and normal vectors, $\mathbf{T}$ and $\mathbf{N}$, are oriented as shown in Fig. 1 , then $\left(T_{u}, T_{v}\right)=\left(N_{v},-N_{u}\right)$. Substituting these relations and formulas

$$
\frac{\partial f}{\partial u}=\frac{\partial f}{\partial s} T_{u}+\frac{\partial f}{\partial N} N_{u} \text { and } \quad \frac{\partial f}{\partial v}=\frac{\partial f}{\partial s} T_{v}+\frac{\partial f}{\partial N} N_{v}
$$

for the derivatives of a function $f$ of $u$ and $v$ into (27), we obtain the following form of the representation formula:

$$
=\int_{\partial \Omega}\left[\left(1-\frac{(\mathbf{q} \cdot \mathbf{T})^{2}}{c^{2}}\right)\left(G \frac{\partial \psi}{\partial N}-\psi \frac{\partial G}{\partial N}\right)+\frac{\mathbf{q} \cdot \mathbf{T} \mathbf{q} \cdot \mathbf{N}}{c^{2}}\left(G \frac{\partial \psi}{\partial s}-\psi \frac{\partial G}{\partial s}\right)+\frac{\mathbf{q} \cdot \mathbf{N}}{c^{2}} G \psi\right] d s
$$


This formula and the boundary conditions derived in Sec. 2 can be used to obtain an integral equation formulation of the problem of subsonic flow around an airfoil. Work on this application is in progress.

4. A fundamental solution. The fundamental solution, $G$, entering the representation formula is chosen to be bounded at $q=0$ and to vanish at $q=q_{\text {max }}$.

With the polar hodograph coordinates $q$ and $\theta$ used as independent variables, Eq. (26) reads:

$$
\frac{\partial^{2} G}{\partial q^{2}}+\frac{1-M^{2}}{q} \frac{\partial G}{\partial q}+\frac{1-M^{2}}{q^{2}} \frac{\partial^{2} G}{\partial \theta^{2}}-\frac{2 c_{0}^{2}}{c^{4}} G=-\frac{\delta\left(q-q^{\prime}\right) \delta\left(\theta-\theta^{\prime}\right)}{q},
$$

where $M=q / c$. Since this equation and the boundary conditions for $G$ are symmetric with respect to the ray $\theta=\theta^{\prime}$, the Fourier series of $G$ has the form

$$
G\left(q, q^{\prime}, \theta-\theta^{\prime}\right)=\sum_{n=0}^{\infty} G_{n} \cos \left(n\left(\theta-\theta^{\prime}\right)\right)=\sum_{n=0}^{\infty} A_{n} Q_{n}(q) \cos \left(n\left(\theta-\theta^{\prime}\right)\right) .
$$

Here $A_{n}$ is a coefficient depending on $q^{\prime}$, and $Q_{n}$ is a solution to the equation

$$
\frac{d^{2} Q_{n}}{d q^{2}}+\frac{1-M^{2}}{q} \frac{d Q_{n}}{d q}-\left(\frac{2 c_{0}^{2}}{c^{4}}+\frac{1-M^{2}}{q^{2}} n^{2}\right) Q_{n}=-\frac{\delta\left(q-q^{\prime}\right)}{\pi q\left(1+\delta_{0 n}\right)},
$$

where $\delta_{0 n}$ is Kronecker's delta.

By the transformation

$$
w=1-\frac{c^{2}}{c_{0}^{2}}=\frac{\gamma-1}{2} \frac{q^{2}}{c_{0}^{2}}
$$

Eq. (32) becomes

$$
\begin{aligned}
\frac{d^{2} Q_{n}}{d w^{2}}+\left[\frac{1}{w}\right. & \left.-\frac{1}{(\gamma-1)(1-w)}\right] \frac{d Q_{n}}{d w} \\
& -\left[\frac{1}{(\gamma-1)(1-w)}+\frac{n^{2}}{4 w}-\frac{n^{2}(\gamma+1)}{4(\gamma+1)}\right] \frac{Q_{n}}{w(1-w)}=-\frac{\delta\left(w-w^{\prime}\right)}{2 \pi\left(1+\delta_{0 n}\right) w},
\end{aligned}
$$

where $w^{\prime}$ is the value of $w$ for $q=q^{\prime}$.

The homogeneous equation corresponding to (34) has regular singularities at $w=0$ and $w=1$. The exponents of the solutions are $n / 2$ and $-n / 2$ at $w=0$ and 1 and $-1 /(\gamma-1)$ at $w=1$. The point at infinity turns out to be a regular singularity, too. Thus, the equation has precisely three singular points, which are all regular singularities, and therefore its solutions can be expressed in terms of hypergeometric functions [16]. Indeed, when its solutions are written as

$$
Q_{n}(q)=w^{-n / 2}(1-w)^{-1 /(\gamma-1)} P_{n}(w),
$$

the equation for $P_{n}$ becomes the hypergeometric equation [16],

$$
w(1-w) \frac{d^{2} P_{n}}{d w^{2}}+\left[1-n-\left(1-n-\frac{1}{\gamma-1}\right) w\right] \frac{d P_{n}}{d w}+\frac{n(n-1)}{2(\gamma-1)} P_{n}=0
$$

In the following, we shall consider two solutions to (36). For the one, denoted by $R_{n}$, the corresponding function $Q_{n}$ is bounded at $w=0$. For the other one, $S_{n}$, the 
corresponding function $Q_{n}$ vanishes at $w=1$. Using standard separation of variables technique, we then find the series in (31) to be

$$
G\left(q, q^{\prime}, \theta-\theta^{\prime}\right)=-\frac{1}{2 \pi(1-w)^{1 /(\gamma-1)}} \sum_{n=0}^{\infty} \frac{R_{n}\left(w_{<}\right) S_{n}\left(w_{>}\right)}{\left(w w^{\prime}\right)^{n / 2}\left(1+\delta_{0 n}\right) C_{n}} \cos \left(n\left(\theta-\theta^{\prime}\right)\right),
$$

where $w_{<}$is the smaller and $w_{>}$is the larger of $w$ and $w^{\prime}$, and the constant $C_{n}$ is determined from the Wronskian:

$$
R_{n} \frac{d S_{n}}{d w}-S_{n} \frac{d R_{n}}{d w}=C_{n} w^{n-1}(1-w)^{1 /(\gamma-1)} .
$$

For $n=0$, Eq. (36) has constant solutions. A solution linearly independent of a constant is found by quadrature, and the resulting integral can be expanded in a power series and integrated termwise. In this way, we get two solutions of the types defined above, namely:

$$
R_{0}(w)=1 \quad \text { and } \quad S_{0}(w)=-(1-w)^{1 /(\gamma-1)}\left[\ln (w)+\sum_{k=1}^{\infty} \frac{(1-w)^{k}}{k((\gamma-1) k+1)}\right] .
$$

For $n=1$, too, the equation has constant solutions and the function $S_{1}=(1-$ $w)^{\gamma /(\gamma-1)}$ is another closed form solution. Therefore, for $n=1$ we define:

$$
R_{1}(w)=1-(1-w)^{\gamma /(\gamma-1)} \quad \text { and } \quad S_{1}(w)=(1-w)^{\gamma /(\gamma-1)}
$$

For $n>1$, the solutions can be expressed in terms of hypergeometric functions defined by the usual formula [16]:

$$
F(a, b ; c ; z)=\frac{\Gamma(c)}{\Gamma(a) \Gamma(b)} \sum_{k=0}^{\infty} \frac{\Gamma(a+k) \Gamma(b+k)}{\Gamma(c+k) k !} z^{k}
$$

We define the following two solutions:

$$
R_{n}(w)=w^{n} F(\alpha+n, \beta+n ; n+1 ; w)
$$

and

$$
S_{n}(w)=\frac{\Gamma(1-\alpha) \Gamma(1-\beta)}{(n-1) ! \Gamma\left(\frac{2 \gamma-1}{\gamma-1}\right)}(1-w)^{\gamma /(\gamma-1)} F\left(\alpha+\frac{\gamma}{\gamma-1}, \beta+\frac{\gamma}{\gamma-1} ; \frac{2 \gamma-1}{\gamma-1} ; 1-w\right) .
$$

Here $\alpha$ and $\beta$ are the two constants:

$$
\left.\begin{array}{l}
\alpha \\
\beta
\end{array}\right\}=-\frac{n}{2}-\frac{1}{2(\gamma-1)} \pm \frac{\left(n^{2}\left(\gamma^{2}-1\right)+1\right)^{1 / 2}}{2(\gamma-1)}
$$

In $S_{n}$, the constant coefficient is chosen so that $S_{n}(0)=1$ (see formula (46) below).

The series for $R_{n}$ converges slowly as the singularity at $w=1$ is approached. The same holds with regard to $S_{n}$ as $w$ approaches 0 . To compute the functions numerically for all $w \in[0,1]$, and to find the constants $C_{n}$ from (38), we construct alternative expressions for 
the two functions using formulac found in [16]. For $R_{n}$, application of formulas (15.3.3) and (15.3.6) in [16] results in the expression:

$$
\begin{aligned}
& R_{n}(w)=\frac{n ! \Gamma\left(\frac{\gamma}{\gamma-1}\right)}{\Gamma(1-\alpha) \Gamma(1-\beta)} F\left(\alpha, \beta ;-\frac{1}{\gamma-1} ; 1-w\right) \\
& \quad+(1-w)^{\gamma /(\gamma-1)} \frac{n ! \Gamma\left(-\frac{\gamma}{\gamma-1}\right)}{\Gamma(\alpha+n) \Gamma(\beta+n)} F\left(\alpha+\frac{\gamma}{\gamma-1}, \beta+\frac{\gamma}{\gamma-1} ; \frac{2 \gamma-1}{\gamma-1} ; 1-w\right),
\end{aligned}
$$

in which the series in (41) can be used. For $S_{n}$, a series which converges rapidly near $w=0$, is found from $(15.3 .11)$ in $[16]$ :

$$
\begin{aligned}
& S_{n}(w) \\
& =(1-w)^{\gamma /(\gamma-1)}\left\{\sum_{k=0}^{n-1} \frac{\Gamma(1-\alpha-n+k) \Gamma(1-\beta-n+k)(n-1-k) !}{\Gamma(1-\alpha-n) \Gamma(1-\beta-n) k !(n-1) !}(-w)^{k}\right. \\
& \left.-\frac{(-w)^{n}}{(n-1) !} \sum_{k=0}^{\infty} \frac{\Gamma(1-\alpha+k) \Gamma(1-\beta+k)}{\Gamma(1-\alpha-n) \Gamma(1-\beta-n) k !(k+n) !} w^{k}\left[\ln (w)-p_{n, k}\right]\right\} .
\end{aligned}
$$

Here

$$
p_{n, k}=\psi(k+1)+\psi(k+n+1)-\psi(1-\alpha+k)-\psi(1-\beta+k),
$$

where $\psi$ has the usual meaning, $\psi(z)=\Gamma^{\prime}(z) / \Gamma(z)$.

Using (42) for $R_{n}$ and (46) for $S_{n}$ at $w=0$ (or (45) for $R_{n}$ and (43) for $S_{n}$ at $w=1$ ), we find the constants $C_{n}$ in (37) and (38) to be:

$$
C_{0}=-1, \quad C_{1}=-\frac{\gamma}{\gamma-1} \quad \text { and } \quad C_{n}=-n \quad \text { for } \quad n>1
$$

The final formula for $G$ is now obtained, when the various expressions derived above are inserted in (37).

5. Numerical evaluation of the fundamental solution. Although we now have at our disposal two series expansions for each function $R_{n}$ and $S_{n}$, one converging rapidly near $w=0$ and the other one near $w=1$, they are not sufficient for accurate, double precision computation for all values of $w$. The reason is that for values of $w$ in the central part of the interval $[0,1]$, the ratio of the sum of any of the infinite series we are considering to its largest term decreases with increasing $n$. For $n$ sufficiently large, it is too small for double precision accuracy $\left(10^{-16}\right)$. To compute $R_{n}$ and $S_{n}$ for all $w$, we use series expansions with expansion points in the interior of the interval $[0,1]$. It is found that if $\left.w_{0} \in\right] 0,1[$, the coefficients of a series:

$$
P_{n}(w)=\sum_{k=0}^{\infty} b_{k}\left(w-w_{0}\right)^{k}
$$


for a solution to Eq. (36) are determined by the following three-term recursion formula:

$$
\begin{aligned}
b_{k+2}=\frac{\left(2 w_{0}-1\right) k-\frac{w_{0}}{\gamma-1}+(n-1)\left(1-w_{0}\right)}{(k+2) w_{0}\left(1-w_{0}\right)} & b_{k+1} \\
+ & \frac{k\left(k-n-\frac{1}{\gamma-1}\right)-\frac{1}{2} n(n-1) \frac{1}{\gamma-1}}{(k+1)(k+2) w_{0}\left(1-w_{0}\right)} b_{k} .
\end{aligned}
$$

In our computations we apply the system of series in the following way. We first use (42), (43), (45), and (46) to compute $R_{n}\left(w_{0}\right)$ and $S_{n}\left(w_{0}\right)$ and the first derivatives, $R_{n}^{\prime}\left(w_{0}\right)$ and $S_{n}^{\prime}\left(w_{0}\right)$, with quadruple precision accuracy (32 digits) at $w_{0}=1 / 3$ and $w_{0}=2 / 3$. The results are thereafter inserted for $b_{0}$ and $b_{1}$ in (50) and the required number of the coefficients $b_{k}$ in (49) are found in double precision. In this way, we are able to evaluate $R_{n}(w)$ and $S_{n}(w)$ with double precision accuracy for $n$ up to 50 and all $w \in[0,1]$ by means of the pair of series of which the expansion point, $w_{0}=0,1 / 3,2 / 3$, or 1 , is closest to $w$.

In the elliptic region (subsonic flow), fundamental solutions diverge toward infinity as the field point $(q, \theta)$ approaches the singular point $\left(q^{\prime}, \theta^{\prime}\right)$. Therefore the rate of convergence of the series in (37) is poor for field points near the singular point. To be able to compute $G$ there also, we need a closed form expression with the same singular behavior near $\left(q^{\prime}, \theta^{\prime}\right)$ as that of a fundamental solution. If $G^{*}$ denotes such an expression, and $G_{n}^{*}$ its $n$th Fourier coefficient, the series in the formula

$$
G=G^{*}+\sum_{n=0}^{\infty}\left(G_{n}-G_{n}^{*}\right) \cos \left(n\left(\theta-\theta^{\prime}\right)\right)
$$

converges more rapidly than the one in $(37)$, if $(q, \theta)$ is close to $\left(q^{\prime}, \theta^{\prime}\right)$.

In order to construct a function $G^{*}$, we rely upon the intuitive assumption that the leading term of a fundamental solution at the singular point is not changed if the coefficients in Eq. (30) are "frozen" at the singular point, i.e., the coefficients are replaced by constants equal to their respective values at that point. With the new independent variables $\xi$ and $\eta$ defined by

$$
\xi=q \cos \left(\theta-\theta^{\prime}\right)-q^{\prime} \quad \text { and } \quad \eta=q \sin \left(\theta-\theta^{\prime}\right)
$$

Eq. (30) reads

$$
\left(1-\frac{\eta^{2}}{c^{2}}\right) \frac{\partial^{2} G}{\partial \xi^{2}}+2 \frac{\xi \eta}{c^{2}} \frac{\partial^{2} G}{\partial \xi \partial \eta}+\left(1-\frac{\left(\xi+q^{\prime}\right)^{2}}{c^{2}}\right) \frac{\partial^{2} G}{\partial \eta^{2}}-2 \frac{c_{0}^{2}}{c^{4}} G=-\delta(\xi) \delta(\eta) .
$$

Freezing the coefficients by putting $\xi=\eta=0$ and $c=c^{\prime} \equiv c\left(q^{\prime}\right)$ and by introducing the new variable $t=\eta\left(1-q^{2} / c^{2}\right)^{-1 / 2}$, we obtain the following inhomogeneous Helmholtz's equation:

$$
\frac{\partial^{2} G^{*}}{\partial \xi^{2}}+\frac{\partial^{2} G^{*}}{\partial t^{2}}-2 \frac{c_{0}^{2}}{c^{\prime 4}} G^{*}=-\left(1-q^{\prime 2} / c^{2}\right)^{-1 / 2} \delta(\xi) \delta(t)
$$

It has the solution

$$
\frac{1}{2 \pi \sqrt{1-q^{\prime 2} / c^{\prime 2}}} K_{0}\left(\frac{\sqrt{2} c_{0}}{c^{\prime 2}} \sqrt{\xi^{2}+t^{2}}\right)
$$


where $K_{0}$ is the modified Bessel function of the third kind, of order 0 . This function could be used for $G^{*}$. However, since its Fourier coefficients cannot be expressed in closed form, they must be evaluated by numerical quadrature and they are difficult to compute accurately if $q$ is close to $q^{\prime}$. Therefore, we use the leading term of (55), only, for $G^{*}$. Expressed in terms of the original variables $q$ and $\theta$, the leading term is

$$
G^{*}=-\frac{1}{4 \pi \sqrt{1-q^{\prime 2} / c^{\prime 2}}} \ln \left[\left(q \cos \left(\theta-\theta^{\prime}\right)-q^{\prime}\right)^{2}+\frac{q^{2} \sin ^{2}\left(\theta-\theta^{\prime}\right)}{1-q^{\prime 2} / c^{\prime 2}}\right] .
$$

Using formulas given in [17], we find the Fourier coefficients of $G^{*}$ to be:

$$
G_{0}^{*}=\ln \left[\frac{\left(a_{1}+\sqrt{a_{1}^{2}-b^{2}}\right)\left(a_{2}+\sqrt{a_{2}^{2}-b^{2}}\right)}{4\left(c^{\prime 2}-q^{\prime 2}\right)}\right]
$$

and

$$
G_{n}^{*}=-\frac{2}{n} \sum_{k=1}^{k=2}\left[\frac{\sqrt{a_{k}^{2}-b^{2}}-a_{k}}{b}\right]^{n}
$$

for $n>0$, where

$$
a_{k}=c^{\prime} \sqrt{c^{2}+q^{2}-q^{\prime 2}}+(-1)^{k}\left(c^{\prime 2}-q^{\prime 2}\right),
$$

and $b=q q^{\prime}$.

The fundamental solution $G$ is now easily computed everywhere in the subsonic region. Graphs of $G$ for various values of $\left(q^{\prime}, \theta^{\prime}\right)$ with $\theta^{\prime}=0$ are presented in Figs. 4 through 9 .

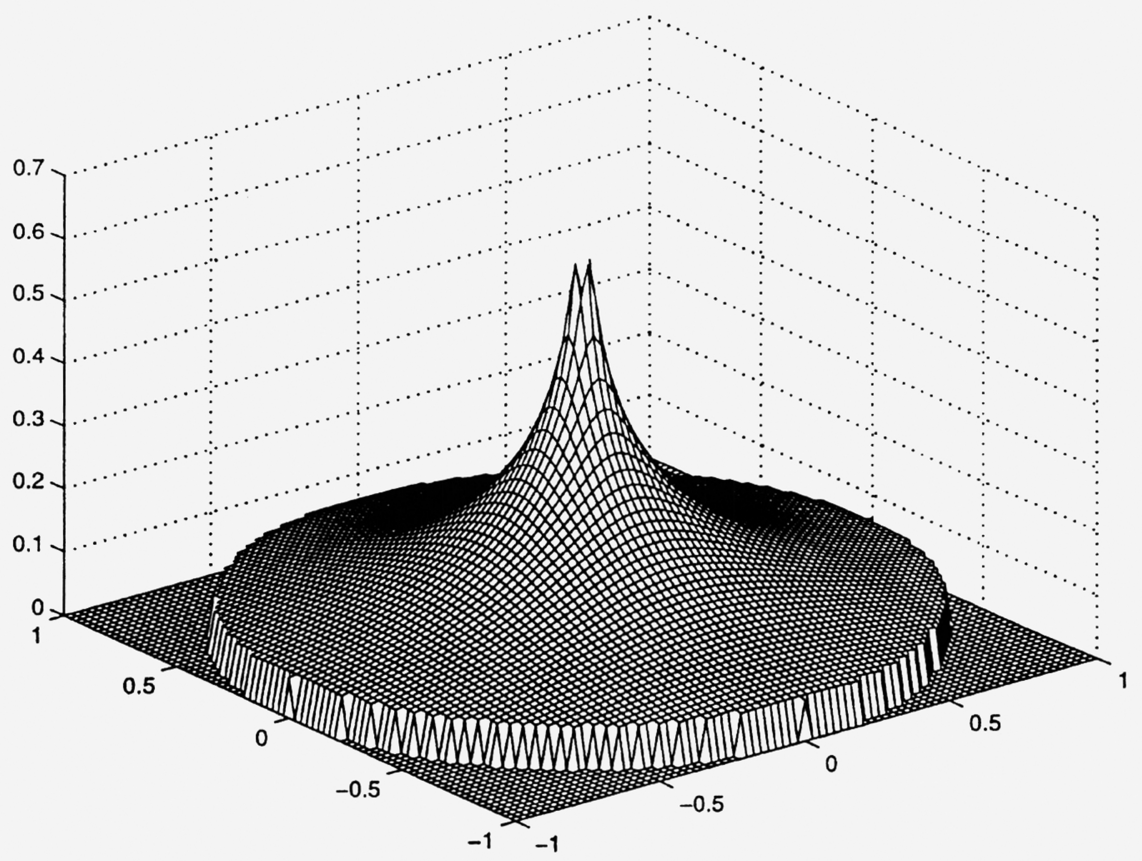

FIG. 4. The fundamental solution in the subsonic region $\left(q<q_{\text {crit }}\right)$ for $q^{\prime}=0$ shown as a function of $u / q_{\text {crit }}$ and $v / q_{\text {crit }}$ 


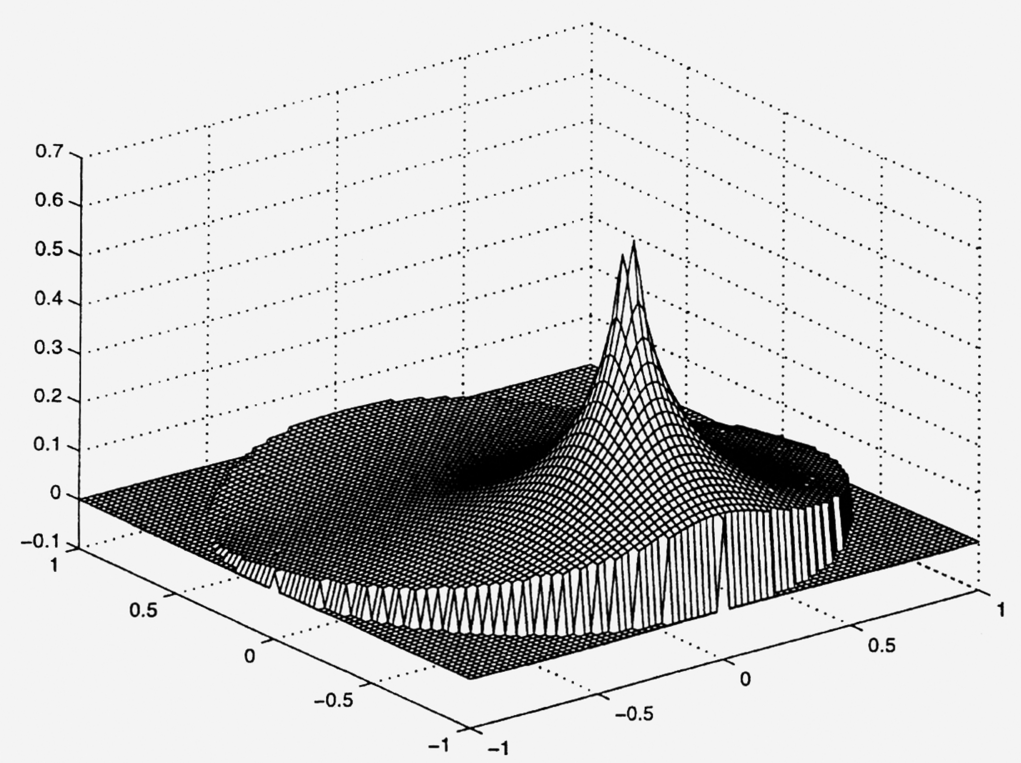

FIG. 5. The fundamental solution in the subsonic region for $q^{\prime}=$ $0.5 * q_{\text {crit }}$ and $\theta^{\prime}=0$

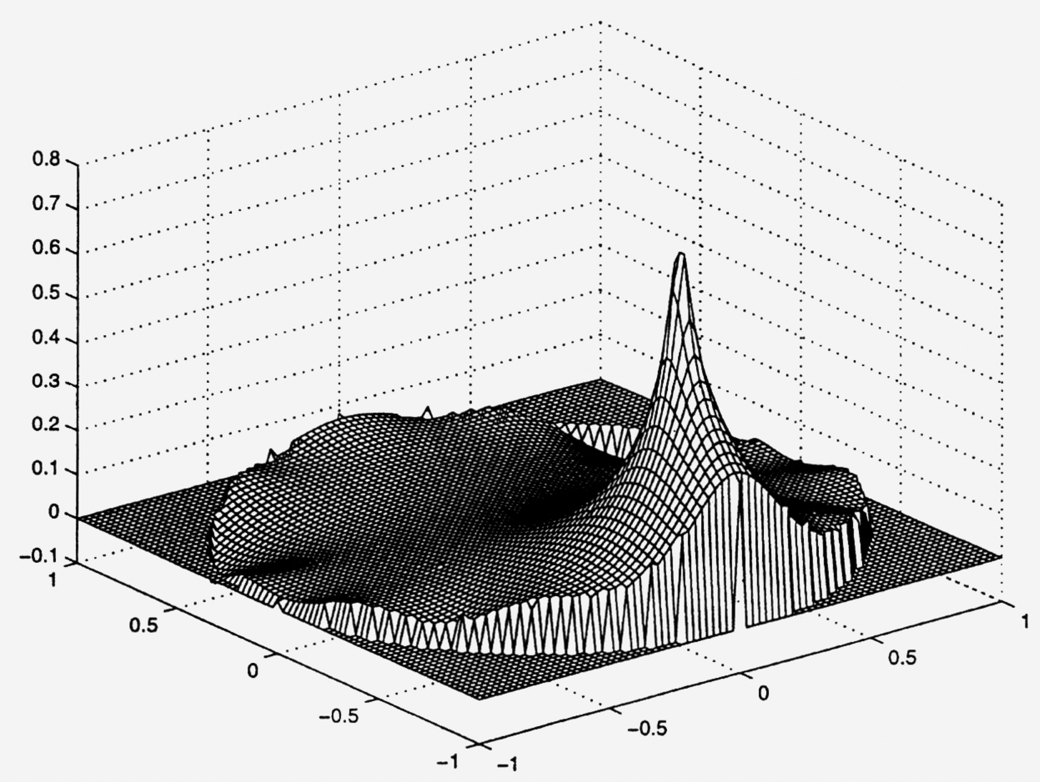

FIG. 6. Same as Fig. 5 except that $q^{\prime}=0.7 * q_{\text {crit }}$ 


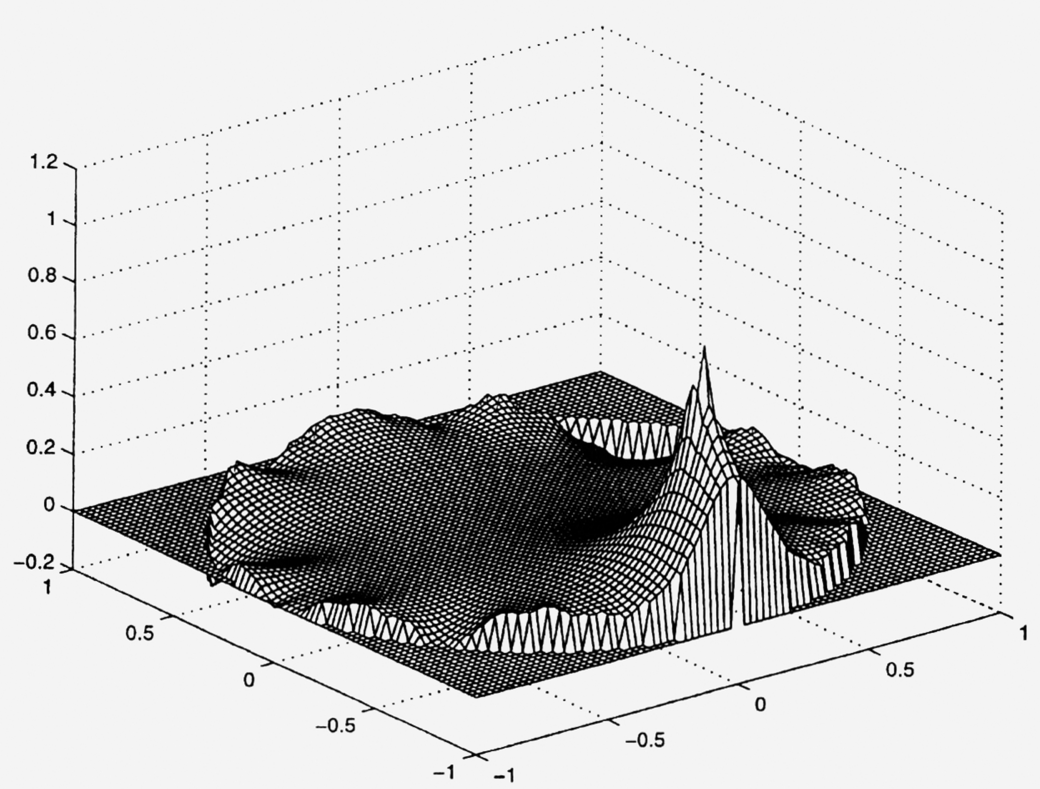

FIG. 7. Same as Fig. 5 except that $q^{\prime}=0.8 * q_{\text {crit }}$

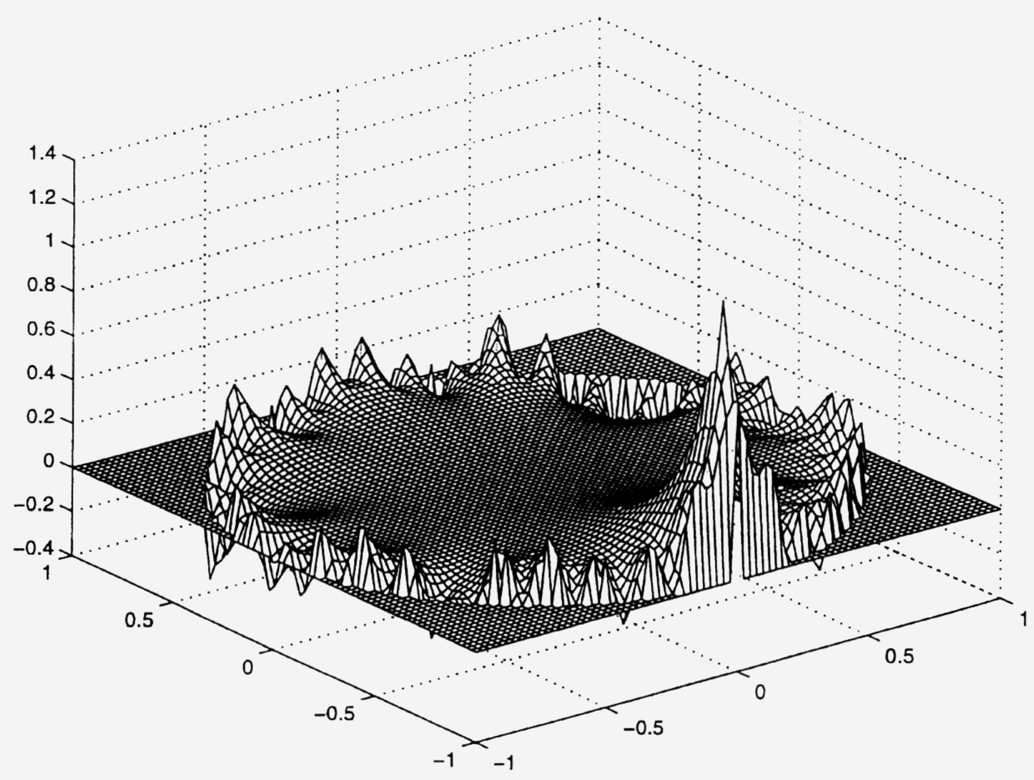

FIG. 8. Same as Fig. 5 except that $q^{\prime}=0.9 * q_{\text {crit }}$ 


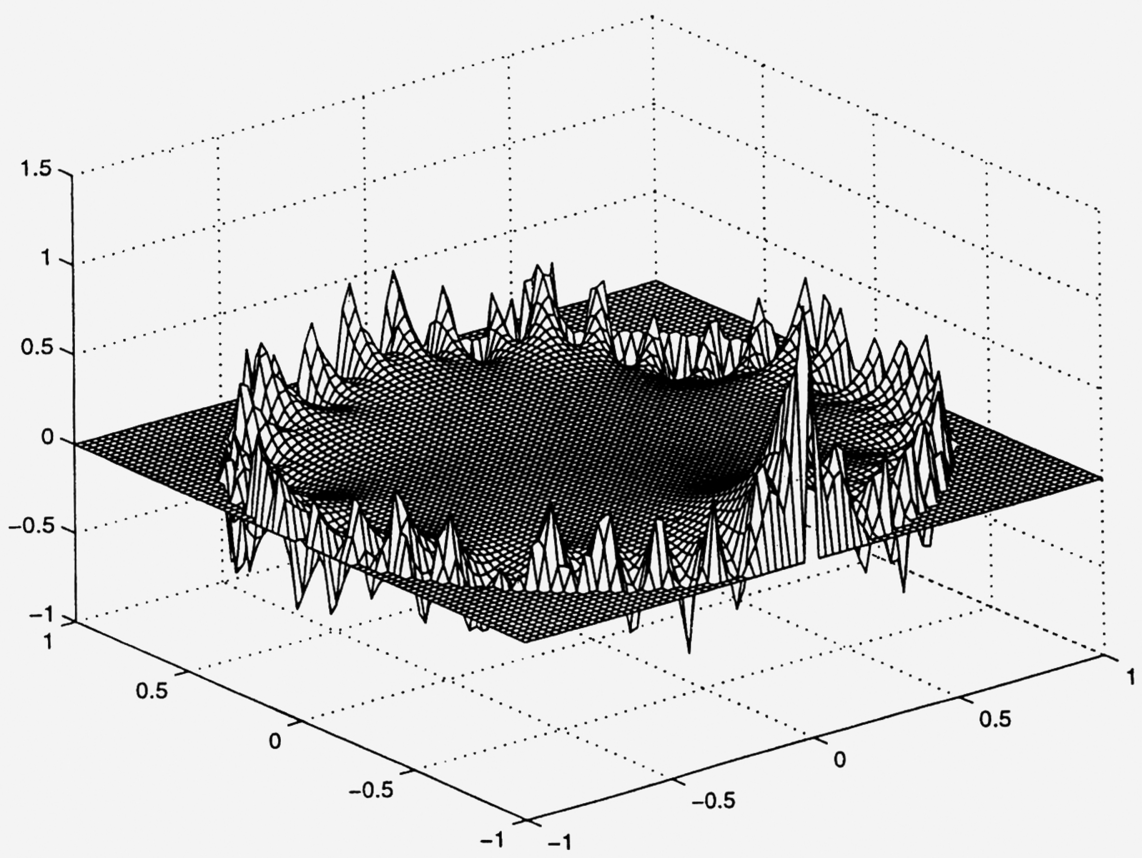

FIG. 9. Same as Fig. 5 except that $q^{\prime}=0.95 * q_{\text {crit }}$

\section{REFERENCES}

[1] L. Morino, Boundary integral equations in aerodynamics, Appl. Mech. Rev., 46, no. 8, 445-466, 1983

[2] C. Pozrikidis, Boundary Integral and Singular Methods for Linearized Viscous Flow, Cambridge University Press, Cambridge, 1992

[3] K. Oswatitsch, Die Geschwindigkeitverteilung bei lokalen Überschallgebiete an flachen Profilen, Z. Angew. Math. Mech., 30, 17-24, 1950

[4] Symposium Transsonicum, K. Oswatitsch, Ed., Springer, Berlin, 1964

[5] Symposium Transsonicum II, K. Oswatitsch and D. Rues, Eds., Springer, Berlin, 1976

[6] M. J. Lighthill, The hodograph transformation in trans-sonic flow. I. Symmetric channels, Proc. Roy. Soc. London Ser. A 191, 323-341, 1947

[7] M. J. Lighthill, The hodograph transformation in trans-sonic flow. II. Auxiliary theorems on the hypergeometric functions $\psi_{n}(\tau)$, Proc. Roy. Soc. London Ser. A 191, 341-351, 1947

[8] M. J. Lighthill, The hodograph transformation in trans-sonic flow. III. Flow round a body, Proc. Roy. Soc. London Ser. A 191, 352-369, 1947

[9] E. Shimborsky, Variational methods applied to the study of symmetric flows in Laval nozzles, Comm. Partial Differential Equations 4, 41-77, 1979

[10] Erik B. Hansen, Stokes flow down a wall into an infinite pool, J. Fluid Mech. 178, 243-256,

[11] Erik B. Hansen and Mark A. Kelmanson, Steady, viscous, free-surface flow on a rotating cylinder, J. Fluid Mech. 272, 91-107, 1994

[12] R. V. Mises, Mathematical Theory of Compressible Fluid Flow, Academic Press, New York, 1958, p. 251

[13] S. Nocilla, Sull'esistenza di flussi transonici continui attorne a profili alari ad arco cerchio, Atti della Academia delle Scienzi di Torino, 94, 796-822, 1960

[14] R. Courant and D. Hilbert, Methods in Mathematical Physics, Interscience Publishers, New York, 1962, Vol. II, p. 336

[15] J. Crank, Free and Moving Boundary Problems, Clarendon Press, Oxford, 1984, chapter 2 
[16] M. Abramowitz and I. Stegun, Handbook of Mathematical Functions, Dover Publications, New York, 9th printing, 1970, pp. 562-564

[17] W. Gröbner and N. Hofreiter, Integraltafel, Zweiter Teil, Bestimmte Integrale, Springer, Wien, 5th ed., 1973, p. 148 